Methods A data collection form was developed using the WHO/ Monash Fatal Injury Surveillance in Mortuaries and Hospitals Manual and was implemented in 4 mortuaries ( 3 urban, 1 rural). Training for project staff, ongoing technical support and monitoring was provided by WHO. The project was led by Forensic Pathologists. Data collection started in 2010, with initial piloting (2010-2011) and implementation after the publication of the manual (2013-present). In 2015, an evaluation was conducted comparing data from the mortuary with data from traffic police, hospital mortality records and with national VR registry.

Results In the initial pilot phase (2010-2011) a total of 112 cases were recorded in the 4 mortuaries. Based on the result of the piloting, the system was revised. Formal implementation started in 2013. By June 2015, a total of 2666 cases were recorded. About two-thirds were from unintentional causes, majority being due to road traffic injuries (RTI). Of the remaining, $6 \%$ were due to suicide, $15 \%$ from assault. Most of the RTI victims were passengers, motorcyclists and pedestrians. Males were over represented in all causes. Most of the deceased were 18-29 years old. Suffocation/hanging was the main mechanism of injury for suicide cases. While guns and blunt objects were the weapons involved for majority of the homicide deaths. There is a high under reporting of RTI deaths in police and VR systems. With recognition of its limitations, the FIS data from the mortuary is being used to support local injury prevention efforts.

Conclusions Compared to other sources, such as police and VR systems, mortuaries are a better surveillance data source for injury deaths, particularly for RTI deaths. In low-mid income countries, it is feasible to implement a standardised fatal injury data collection process at both rural and urban mortuaries. Local ownership of the system is key for sustainability.

\section{VALIDATING WISQARS ESTIMATES OF THE INCIDENCE AND COST OF US INJURIES: A TALE OF TWO DATASETS}

1,2 Ted Miller, 'Bruce Lawrence, ${ }^{1}$ Rebecca Spicer. 'Pacific Institute for Research and Evaluation, USA; ${ }^{2}$ Centre for Population Health Research, Curtin University, Australia

\subsection{6/injuryprev-2016-042156.163}

Background Both the US Centres for Disease Control and Prevention's WISQARS system and the Healthcare Cost and Utilisation Program (HCUP) provide sample-based national estimates of injury survivors treated in hospital emergency departments and released (ED cases) or admitted. The WISQARS sample is much smaller but also more quickly available and more fully causecoded than the HCUP data. For admissions, HCUP weights are quite accurate, computed from a near-census. We compared estimated medical and work loss costs from the two systems.

Methods We applied an updated version of essentially the same published injury cost model to both datasets. The HCUP model combined HCUP data on hospital payments per case with estimates by International Classification of Diseases, $9^{\text {th }}$ EditionClinical Modification diagnosis of associated professional fees and lifetime medical costs post-discharge. Work loss costs were estimated from length of stay if admitted and data by diagnosis group on mean days of work loss and the probability of permanent work-related disability. We used 2010 HCUP incidence data to collapse the HCUP costs into WISQARS diagnosis codes.

Results Both data sets estimated 27 million ED cases annually in 2012/13, with annual costs estimated at $\$ 165$ billion using HCUP data and $\$ 167$ billion using WISQARS data. For admitted injuries, WISQARS estimates of 3 million cases costing \$290 billion exceeded HCUP "gold standard" estimates of 2.7 million (11.5\% fewer) and $\$ 245$ billion (19\% less). WISQARS reported admitted injury trends by cause that HCUP suggested were spurious.

Conclusions WISQARS provides adequate injury incidence and cost estimates for surveillance. Its sample is too thin to support trend analyses by cause or for admissions and its admissions estimates should be replaced or reweighted with HCUP data as they become available. For admitted injuries, the sample of 66 hospitals underlying the WISQARS estimates warrants expansion.

\section{ASSESSING VIOLENCE AND INJURY SURVEILLANCE IN THE CARIBBEAN}

Glennis Andall-Brereton, William Mueller. Caribbean Public Health Agency (CARPHA) Trinidad and Tobago

\subsection{6/injuryprev-2016-042156.164}

Background The Caribbean Region experiences high levels of violence and injury. Limited research is available on the status of existing violence and injury prevention (VIP) efforts and surveillance systems.

Methods The Caribbean Public Health Agency undertook a survey to gather evidence of VIP in 24 member countries. The questionnaire on VIP was developed using available literature on injury surveillance systems. The survey was administered to national epidemiologists and non-communicable disease focal points in October 2015 using an online tool, "Survey Monkey". Questions were asked about the availability in each country of VIP representative surveys, policies, action plans, laws, victim support services, and surveillance systems.

Results Nineteen (79\%) countries completed the VIP survey. Only three $(16 \%)$ countries confirmed having undertaken a nationally representative survey. Twelve (63\%) had not developed a national policy and 14 (74\%) had not implemented an action plan on VIP. Each country reported the existence of VIP laws and offered victim support services, though average enforcement ranged from $40-79 \%$. Nine (47\%) countries indicated using an injury registry and 15 (79\%) reported collecting injury data with in-patient records, mainly from public hospitals. All 19 countries confirmed that records of reported violent incidents were maintained by the police. Unique identifiers were generally lacking in registries and police systems. Only four (44\%) countries with registries, ten $(67 \%)$ countries with in-patient injury data, and 12 $(63 \%)$ countries with police records indicated sharing data with other organisations.

Conclusions Each country reported some level of injury surveillance system; however, such systems should be harmonised to produce more complete baseline data. The use of unique identifiers is required to reduce duplication and effectively link surveillance systems.

\section{THE SWEDISH NATIONAL INFORMATION SYSTEM FOR TRAFFIC ACCIDENTS AND INJURIES; EXPERIENCES FROM THE IMPLEMENTATION PROCESS}

Tomas Fredlund, Monica Frank. The Swedish Transport Agency

10.1136/injuryprev-2016-042156.165

Background The process towards a new Swedish information system was launched in 1993 when the Swedish Road 